\title{
Oral paclitaxel with encequidar compared to intravenous paclitaxel in patients with advanced cancer: a randomised crossover pharmacokinetic study.
}

Christopher Jackson ${ }^{1}$, C Hung ${ }^{2}$, Eva Segelov ${ }^{3}$, Paula Barlow ${ }^{4}$, Hans Prenen ${ }^{5}$, Blair McLaren $^{6}$, Noelyn Hung ${ }^{7}$, Katriona Clarke ${ }^{8}$, Tsu-Yi Chao ${ }^{9}$, Ming-Shen Dai ${ }^{10}$, Hsien-Tang Yeh $^{11}$, David Cutler ${ }^{12}$, Doug Kramer ${ }^{12}$, Jimmy He ${ }^{12}$, Jay Zhi ${ }^{12}$, Wing-Kai Chan ${ }^{12}$, Rudolf $\mathrm{Kwan}^{12}$, and Sanjeev Deva ${ }^{4}$

${ }^{1}$ University of Otago Medical School

${ }^{2}$ Affiliation not available

${ }^{3}$ Monash University SCS at Monash Health

${ }^{4}$ Auckland District Health Board

${ }^{5}$ University Hospital Antwerp

${ }^{6}$ Southern Blood and Cancer Service

${ }^{7}$ University of Otago

${ }^{8}$ Capital and Coast District Health Board

${ }^{9}$ Taipei Medical University Shuang Ho Hospital Ministry of Health and Welfare

${ }^{10}$ Tri-Service General Hospital

${ }^{11}$ Lo-Hsu Medical Foundation Lotung Poh-Ai Hospital

${ }^{12}$ Athenex Inc

January 4, 2021

\begin{abstract}
Background and purpose: Paclitaxel is a widely used anti-neoplastic agent but has low oral bioavailability due to gut extrusion by P-glycoprotein (P-gp). Oral paclitaxel could be more convenient, less resource intensive, and more tolerable than intravenous administration. Encequidar (HM30181A) is a novel, minimally absorbed gut specific P-gp inhibitor. We tested whether administration of oral paclitaxel with encequidar (oPac+E) achieved comparable AUC to intravenous paclitaxel (IVP) 80mg/m2. Experimental approach: We conducted a multi-centre randomised crossover study with two treatment periods. Patients (pts) with advanced cancer received either oral paclitaxel $615 \mathrm{mg} / \mathrm{m} 2$ divided over three days and encequidar $15 \mathrm{mg}$ orally one-hour prior, followed by IVP $80 \mathrm{mg} / \mathrm{m} 2$, or the reverse sequence. PK blood samples were taken up to day 9 for oPac $+\mathrm{E}$ and day 5 for IVP. Key Results: 42 pts were enrolled; 35 completed both treatment periods. AUC0-[?]was 5033.5 +/- $1401.1 \mathrm{ng} . \mathrm{h} / \mathrm{mL}$ for oPac+E and 5595.9 +/- $1264.1 \mathrm{ng} . \mathrm{h} / \mathrm{mL}$ with IVP. The geometric mean ratio (GMR) for AUC was 89.5\% (90\% CI 83.9-95.5). Mean absolute bioavailability of oPac+E was $12 \%$. PK parameters did not change meaningfully after 4 weeks administration of oPac $+\mathrm{E}$ in an extension study. G3 treatment emergent adverse events occurred in 7 (18\%) pts with oPac+E and 2 (5\%) with IVP. $75 \%$ of pts preferred oPac+E over IVP. Conclusion and Implications: GMR for AUC was within the predefined acceptable range of $80 \%-125 \%$ for demonstrating equivalence. oPac+E is tolerable and there is no evidence of $\mathrm{P}$-gp induction with repeat administration. With further study, oPac $+\mathrm{E}$ is a candidate to replace IVP.
\end{abstract}

\section{Introduction}


Taxanes are a class of commonly used chemotherapy compounds, originally identified from Taxus plants. The classical taxanes docetaxel and paclitaxel are widely used anti-neoplastic agents with activity in multiple solid tumours including breast [1], ovarian [2], lung [3], and gastric cancer [4], as well as Kaposi and angiosarcoma [5]. Newer taxanes include cabazitaxel [6] and the nanoparticle-bound nab-paclitaxel [7]. The principal mechanism of action is thought to be disruption of microtubule function. Microtubules are essential for cell division and taxanes stabilize the GDP-bound tubulin in the microtubule, causing inhibition of cell division. Paclitaxel was first discovered in 1963 as part of an National Cancer Institute funded drug candidate screening programme, with activity in mouse models noted in 1978 [8, 9]. Its wide utility has resulted in it being listed on the WHO essential medicines list. The development of paclitaxel was initially hindered by insolubility in water, thus it is administered with the formulation vehicle cremophor EL (in a 1:1 mixture with dehydrated ethanol), which greatly increases the rate of hypersensitivity reactions. Paclitaxel is administered in a variety of dosage regimens as monotherapy or combination, frequently as a weekly infusion at a dose of $80 \mathrm{mg} / \mathrm{m}^{2}$ [10]. Patients are required to have repeated venepuncture, and the schedule uses expensive and often scare hospital infusion resources as well as valuable patient time.

Oral administration may improve convenience and have the potential to reduce costs. During COVID-19 global pandemic, oncologists are substituting oral for intravenous agents to reduce the number of patients' clinic visits and the inherent risks of exposure to SARS-CoV-2, without compromising oncological outcome[11] (Hence, whenever possible, utilization of oral therapy regimens is recommended instead of intravenous anticancer therapies, if considered equivalent[12].

Paclitaxel has low oral bioavailability due to structural instability in the gastrointestinal tract, active extrusion from enterocytes by p-glycoprotein (P-gp) and first pass metabolism by the liver enzymes CYP3A4 and CYP2C8[13] [14] [15] [16]. Paclitaxel absorption is enhanced in P-gp and CYP knockout mice. Preclinical studies have evaluated combinations of Cyclosporine A, a known P-gp inhibitor and substrate for CYP3A4, and oral paclitaxel and showed a 13 fold increase in the oral bioavailability in mice[17]. Subsequently, Phase 1 and 2 studies investigated oral paclitaxel combination with cyclosporine A and showed promising results[16, 18], however repeated use of cyclosporine A could also lead to serious adverse events such as hypertension and nephrotoxicity Investigation into other P-gp inhibitors such as elacridar and GF120918 have been commenced but not developed for routine use in the clinical setting.

Therefore more specific P-gp inhibitors have been developed such as HM30181A (encequidar; Hanmi Pharmaceutical; Seoul, Korea) [19, 20]. Encequidar is a novel, poorly absorbed, potent, selective gut specific p-glycoprotein inhibitor. Due to low oral bioavailability, the effects of Encequidar are limited to the intestinal enterocyte. In healthy volunteer studies, encequidar was well tolerated with no serious adverse effects at doses ranging from $180 \mathrm{mg}$ to $900 \mathrm{mg}$ in a single dose, and $60 \mathrm{mg}$ to $360 \mathrm{mg}$ doses daily for 5 days, with the maximum tolerated dose not reached.[21]

In a phase 1 study 24 patients received escalating doses of oral paclitaxel with encequidar (oPac $+\mathrm{E}$, previously also known as Oraxol) to determine the maximum tolerated dose (DLTs)[22]. In this study, the dose of paclitaxel was escalated from 60 to $420 \mathrm{mg} / \mathrm{m}^{2}$ and the dose of encequidar from 30 to $210 \mathrm{mg} / \mathrm{m}^{2}$ (half the dose of paclitaxel). The drugs were administered on days 1,8 and 15 of each 28 day cycle. No premedication for hypersensitivity was delivered. Only one patient experienced a DLT (grade 3 neutropenia) at $240 \mathrm{mg} / \mathrm{m}^{2}$ of paclitaxel. The MTD was not reached in this study but maximum plasma concentration of paclitaxel was obtained at a dose level of $300 \mathrm{mg} / \mathrm{m}^{2}$.

In another phase I / II study with oPac+E, paclitaxel was orally administered at escalating doses (90, 120 or $\left.150 \mathrm{mg} / \mathrm{m}^{2}\right)$ with a fixed dose $(15 \mathrm{mg} /$ day $)$ of encequidar[23]. oPac+E was administered 6 times per cycle (day $1,2,8,9,15$ and 16) every 4 weeks. In the phase 1 component of the study $(\mathrm{n}=10)$, the MTD could not be determined but based on toxicity and pharmacokinetic data, the recommended phase 2 dose of oral paclitaxel in this 2 consecutive day schedule was determined to be $150 \mathrm{mg} / \mathrm{m}^{2}$ per day.

In contrast to a dose escalation approach to determining optimal dosing schedule, we adopted a pharmacokinetic (PK) driven approach comparing the PK profile of sequential oral doses of oPac $+\mathrm{E}$ to the profile of IV 
paclitaxel. In a pilot pharmacokinetic study, we enrolled patients with advanced cancer who were scheduled to receive $\mathrm{oPac}+\mathrm{E}$ and compared IV administration of paclitaxel with oPac+E[24]. Three cohorts were enrolled with escalating oral paclitaxel doses of $270 \mathrm{mg} / \mathrm{m}^{2}$ (6 patients), $274 \mathrm{mg} / \mathrm{m}^{2}$ (2 patients) or $313 \mathrm{mg} / \mathrm{m}^{2}(2$ patients) daily over two consecutive days, preceded by Encequidar $15 \mathrm{mg}$ (fixed dose). With a two-day dosing schedule saturation at $274 \mathrm{mg} / \mathrm{m}^{2}$ was observed. PK modelling predicted a three-day schedule of $205 \mathrm{mg} / \mathrm{m}^{2}$ per day could achieve bioequivalence between oPac+E and IV paclitaxel $80 \mathrm{mg} / \mathrm{m}^{2}$

To test this hypothesis, we undertook a multicenter, open label, 2 stage study with a 2-treatment period crossover design to test whether oPac+E achieved comparable exposure by AUC to IV paclitaxel $80 \mathrm{mg} / \mathrm{m}^{2}$. We also undertook an extension study to test safety of repeated administration of oPac+E, with repeat PK after 4 weeks administration to test whether potential accumulation or P-gp/CYP induction occurred that may affect systemic concentrations and potentially diminish efficacy.

\section{Methods}

\section{Study oversight}

The study was listed on the Australian New Zealand Clinical Trials Registry website (15/STH/87), approved by the independent ethics committee at each participating institution and conducted in accordance with standard operating procedures of the Sponsor which were designed to ensure adherence to Good Clinical Practice (GCP) guidelines. All the patients provided written informed consent before enrolment into the study.

\section{Eligibility criteria}

Participants were required to have advanced cancer; be scheduled to receive weekly paclitaxel $80 \mathrm{mg} / \mathrm{m}^{2}$ as monotherapy or in combination with other agents; provide written informed consent; age $>/=18$, Eastern Cooperative Oncology Group Performance Status (ECOG PS) 0 or 1, and have life expectancy of 3 months or more. Other key inclusion criteria were: adequate bone marrow reserve $(\mathrm{Hb}>/=90 \mathrm{~g} / \mathrm{L}$, neutrophil count $>/=1.5 \times 10^{9} / \mathrm{L}$, platelet count [?]100 $\times 10^{9} / \mathrm{L}$ ), hepatic reserve (total bilirubin of [?]20 $\mu \mathrm{mol} / \mathrm{L}$ or, for subjects with liver metastasis, [?]30 $\mathrm{mol} / \mathrm{L}$; alanine aminotransferase (ALT) [?]3 $\times$ upper limit of normal (ULN) or, for subjects with liver metastasis, [?] $5 \mathrm{x}$ ULN; alkaline phosphatase (ALP) [?]3 $\mathrm{x}$ ULN or, for subjects with liver or bone metastasis, [?] $5 \times$ ULN, gamma glutamyl transferase (GGT) $<10 \times \mathrm{ULN}$ ), and adequate renal function (calculated creatinine clearance $>50 \mathrm{ml} / \mathrm{min}$ by Cockcroft-Gault, or $\mathrm{Cr}<177 \mu \mathrm{mol} / \mathrm{L}$ ), willing to fast $8 \mathrm{~h}$ prior and 4 hour post dose, and refrain from alcohol and caffeine. Concomitant inducers or inhibitors of P-gp or CYP $3 \mathrm{~A} 4$ or $2 \mathrm{C} 9$ were prohibited within 2 weeks of study medicine.

\section{Study design and treatment}

The was a multi-centre, open-label study with randomised cross-over design. There were two treatment periods: in the $\mathrm{oPac}+\mathrm{E}$ treated group, patients were to receive encequidar $15 \mathrm{mg}$ daily for three days a week and oral paclitaxel $615 \mathrm{mg} / \mathrm{m}^{2}$ divided over 3 days with rounding to the nearest $30 \mathrm{mg}$ capsule. At the completion of the two treatment periods, patients were eligible to return to usual care with weekly paclitaxel, or enter into an extension study of oPac+E three days per week at the dose administered in the current study. The encequidar tablet was administered 1 hour before the oral paclitaxel capsules. In the IV paclitaxel group, patients received weekly Taxol@ $80 \mathrm{mg} / \mathrm{m}^{2}$ IV infused over one hour. Patients were randomised by computer generated central randomization scheme to receive oPac+E followed by IV Taxol, or the reverse sequence. Subjects were instructed to fast for at least 8 hours before and 4 hours after oPac $+\mathrm{E}$ dosing.

Intravenous paclitaxel was administered according to standard local practice. oPac+E was administered in an inpatient clinical research unit. Patients receiving IV PAC were administered standard prophylactic steroids and antihistamines. No premedication was allowed before the first dose of oPac $+\mathrm{E}$; in the follow-up extension study premedication was permitted before subsequent doses for hypersensitivity reactions and/or nausea or vomiting. Intensive blood sampling was undertaken for the first 3 days while on oPac+E, with samples taken pre-dose, after dosing $(0.25,0.5,0.75,1,1.25,1.5,2,3,4,6,8,12$, and 18 hours), on Day 2 to Day 5 or 9 to Day 12, samples were taken predose, after dosing $(0.25,0.5,0.75,1,1.25,1.5,2,3,4,6,8,12$, 
18, 24, 32, 48, 56, 72, 96, 120 and 144 hours). With Taxol@ administration blood sampling was performed pre-dose, during infusion $(2,5,8,12,20,40$, and 60 minutes), after infusion $(0.25,0.5,0.75,1,2,3,4,6$, 8,12 , and 24 hours, then at 32, 48, 56, 72 and 96 hours post dose. Standard baseline investigations were performed including physical exam, ECG, pregnancy test in pre-menopausal female subjects, haematology and biochemistry samples, and urinalysis. After completing both treatment periods patients were asked which treatment they preferred. Adverse events were collected and graded according to CTCAE 4.03, and causality assessed by investigators.

\section{Bioanalytical Methods}

Plasma concentrations of paclitaxel were measured using a validated liquid chromatography/mass spectrometry/ mass spectrometry assay with a lower limit of quantification of $2.5 \mathrm{ng} / \mathrm{mL}$. The calibration curve was prepared by spiking the drug free plasma with known concentrations of paclitaxel $(2.5-2560.0 \mathrm{ng} / \mathrm{ml})$ consisting of a blank sample (matrix sample processed without analyte or internal standard), a zero sample (matrix sample processed without analyte but with internal standard) and twelve non-zero plasma samples, including the LLOQ. Weighted linear regression (1/Y2) was used for the determination of paclitaxel. The back calculated concentrations of the calibration standards should be within $\pm 15 \%$ of the nominal value except for the LLOQ for which it should be within $\pm 20 \%$. The coefficient of determination (R2) of calibration curve exceeded $0.99(\mathrm{r}>0.99)$.

The ability to dilute sample originally above the upper limit of the standard curve has been demonstrated by accuracy and precision parameters during paclitaxel method validation. For those samples above highest standard should be diluted half with drug free plasma and analysed duplicating (reanalysis). Samples below the LLOQ $(2.5 \mathrm{ng} / \mathrm{ml})$ were not included in the data results.

\section{Data and Statistical analysis}

Statistical analyses were reported using summary tables, graphs, and data listings. Continuous variables were summarized using the N, mean, SD, median, minimum, and maximum. Summaries of PK parameters also included the geometric mean and the coefficient of variation. Categorical variables were summarized by counts and by percentage of subjects in corresponding categories. All raw data obtained from the electronic case report form (eCRF), as well as any derived data, were included in data listings.

\section{Pharmacokinetic Analyses}

All subjects who received both study treatments, completed scheduled posttreatment PK evaluations, and were protocol compliant were included in the PK analysis set. Subjects who vomited within twice the median $\mathrm{T}_{\max }$ were excluded from the primary analysis.

Paclitaxel plasma concentrations were normalized to $615 \mathrm{mg} / \mathrm{m}^{2}$ for oPac+E and $80 \mathrm{mg} / \mathrm{m}^{2}$ for IV paclitaxel. Pharmacokinetic and statistical analyses were based on normalized plasma concentrations. Plasma concentrations for paclitaxel were analysed to determine the following PK parameters by noncompartmental analysis using plasma concentration time data for oral and IV paclitaxel: $\mathrm{AUC}_{0-[?]}$ (primary endpoint) as well as $\mathrm{C}_{\max }, \mathrm{AUC}_{0-\mathrm{t}}, \mathrm{T}_{\max }$, and $\mathrm{t}_{1 / 2}$ (secondary endpoints).

The equivalence of the extent of exposure was determined by comparing the $\mathrm{AUC}_{0-[?]}$ of the selected dose of oral paclitaxel (as oPac+E) (administered over 3 consecutive days) to the $\mathrm{AUC}_{0-[?]}$ of IV paclitaxel.

The primary PK parameters were compared between IV paclitaxel (reference) and oral paclitaxel (test) formulations. Analysis of variance (ANOVA) was performed $(\alpha=0.05)$ on the untransformed and $\log 10$ transformed $\mathrm{PK}$ parameters $\mathrm{C}_{\max }, \mathrm{AUC}_{0-\mathrm{t}}$, and $\mathrm{AUC}_{0-[?]}$ for paclitaxel. The ANOVA model included sequence, subjects nested within the sequence, period, and formulation as factors. The significance of the sequence effect was tested using the subjects nested within the sequence as the error term. Two-sided $90 \%$ CIs for the log transformed ratio of test/reference of the least squares means obtained from the ANOVA for $\mathrm{C}_{\max }, \mathrm{AUC}_{0-\mathrm{t}}$, and $\mathrm{AUC}_{0-[?]}$ were estimated. Equivalence was to be concluded if the $90 \% \mathrm{CI}$ of the ratio 
(oral paclitaxel $615 \mathrm{mg} / \mathrm{m}^{2}$ over 3 consecutive days $[\mathrm{oPac}+\mathrm{E}] / 80 \mathrm{mg} / \mathrm{m}^{2} \mathrm{IV}$ paclitaxel) of the least square means from the ANOVA of the log-transformed $\mathrm{AUC}_{0-[?]}$ was within the $80 \%$ to $125 \%$ acceptance range.

\section{Safety Analyses}

For treatment emergent adverse events (TEAEs), verbatim terms were mapped to preferred terms (PTs) and system organ classes (SOCs) using the Medical Dictionary for Regulatory Activities (MedDRA; version 20.0); CTCAE v4.03 was used to grade severity. Incidences of TEAEs were displayed by SOC and PT, and summarized by treatment, treatment period, and treatment sequence. Treatment emergent AEs and SAEs were listed with start and end dates (unless ongoing), severity, relationship to study drug, study drug action taken, and outcome by subject. Laboratory evaluations were summarized and evaluated for markedly abnormal values based on the normal range; shift tables from baseline to posttreatment were also provided for select parameters. Results from ECGs and ECOG Performance Status were summarized. Vital signs, physical examination findings, and treatment preference were listed by subject. Treatment preference was summarized and listed.

Sample Size Rationale

It was planned to enrol an initial cohort of up to 6 patients with an oPac+E dosing regimen consisting of oral encequidar $15 \mathrm{mg}$ plus an oral paclitaxel dose of $615 \mathrm{mg} / \mathrm{m}^{2}$ divided over 3 consecutive days taken in once daily doses. An interim analysis of pharmacokinetic $(\mathrm{PK})$ data was conducted to determine the intrasubject variability and geometric mean ratio $(\mathrm{GMR})$ for $\mathrm{AUC}_{0-[?]}$. If it appeared likely that the selected regimen could meet the criteria for $\mathrm{BE}$ based on $\mathrm{AUC}_{0-[?]}$, additional subjects would be enrolled in Stage 2 at this dose. Based on this pre-planned interim analysis, an additional 34 evaluable subjects were enrolled into Stage 2. A total sample size of 40 evaluable subjects was projected to provide $90 \%$ power for the $90 \%$ CI of the geometric mean ratio for $\mathrm{AUC}_{0-[?]}$ to fall in the range of $80 \%$ to $125 \%$.

\section{Protocol amendments}

During the study, the protocol was amended to allow extended treatment delay or interruption between treatment periods to permit recovery from toxicity or other reason, lowered $\mathrm{Hb}$ eligibility threshold from $100 \mathrm{~g} / \mathrm{L}$ to $90 \mathrm{~g} / \mathrm{L}$, and added inclusion criteria of gamma glutamyl transferase $<10 \times$ ULN to demonstrate adequate liver function based on emerging safety data from other ongoing studies of oPac+E. Other changes were non-substantive.

\section{Results}

\section{Recruitment}

The study was conducted at 3 sites in New Zealand, 1 site in Australia, and 3 sites in Taiwan between 26 Aug 2015 and 27 Mar 2019. 6 patients were recruited at one site (Dunedin, NZ) into the first cohort. Based on interim safety and PK data, it was elected to recruit 34 further patients at the same dose schedule.

In total, a total of 42 subjects were randomised, two participants experienced AEs prior to receiving any study drug and were withdrawn, and 40 subjects received study drug (37 received both OPE and IV paclitaxel, 2 received OPE only, and 1 received IV paclitaxel only). Two patients who received both treatments collected IV PK samples only up to the end of infusion and hence were incomplete regarding PK assessment. As such, 40 participants were considered for the safety analysis, and 35 were included for the PK analysis set (see fig 1 for further detail).

\section{Baseline characteristics}

The median age was 59 years (range 32-78), with the majority of subjects being female $65 \%$ and Caucasian $73 \%$. Demographic and tumour characteristics are listed in Table 1.

PK data 
Derived paclitaxel PK parameters are summarized in Tables 2 and 3 and Figure 2. Following administration of paclitaxel, mean time to peak concentration was approximately 1 hour post oral and IV dosing (figure 2 ). Mean terminal $t_{\frac{1}{2}}$ was longer with oral dosing (43 hours) than after IV administration ( 26 hours). Mean $\mathrm{C}_{\max }$ following oral administration of paclitaxel was one-seventh of that following IV administration. For oral administration, $\mathrm{AUC}_{0-[?]}$ was 5033.5 +/- $1401.1 \mathrm{ng} . \mathrm{h} / \mathrm{mL}$ compared to 5595.9 +/- $1264.1 \mathrm{ng} . \mathrm{h} / \mathrm{mL}$ with IV. The intrasubject coefficient of variation was $16.1 \%$. Based on log transformed data, the geometric mean ratio (GMR) for AUC was 89.5\% (90\% CI 83.9-95.5). The $90 \%$ CI was within the predefined acceptable range of $80 \%$ to $125 \%$ for demonstrating bioequivalence. The mean absolute bioavailability of oral compared to IV paclitaxel was $12 \%$. There was no difference in bioavailability or AUC by Asian compared to European ethnicity (Figure 3).

\section{Toxicity}

Toxicity is summarised in table 4. At least 1 treatment emergent adverse event (TEAE) was reported in 38 (95\%) Safety Analysis Set subjects. More subjects reported TEAEs during oPac+E treatment (36 [92\%] subjects) than during IV paclitaxel treatment (29 [76\%]). Grade 3 TEAEs were reported in $8(20 \%)$ subjects, inclusive of $7(18 \%)$ subjects during oPac $+\mathrm{E}$ treatment and $2(5 \%)$ subjects during IV paclitaxel treatment. Serious TEAEs were reported in $6(15 \%)$ subjects and were judged treatment-related by the Investigator in $2(5 \%)$ subjects, both of whom were during oPac+E treatment. Three $(8 \%)$ subjects had at least 1 TEAE resulting in discontinuation of study drug $(1[3 \%]$ during oPac+E treatment and $2[5 \%]$ during IV paclitaxel treatment) and $2(5 \%)$ subjects discontinued from the study due to a TEAE (both during IV paclitaxel treatment). One subject died 26 days after her last dose of on-study IV paclitaxel.

\section{Patient preference}

Patients who completed both IV and oral dosing were asked which treatment they preferred after completion of both treatment periods. Thirty $(75 \%)$ subjects preferred oPac+E treatment over IV paclitaxel, while IV paclitaxel was preferred over oPac+E in $6(15 \%)$ subjects. No treatment preference was reported in the remaining 4 subjects.

\section{Discussion and Conclusions}

Paclitaxel is a widely used anti-neoplastic agent and weekly schedules are now in common usage. This however results in considerable consumption of health care resources, and precious patient time. Oral formulations have several potential advantages but their development is complex. Paclitaxel is poorly absorbed due to active extrusion by P-gp and undergoes extensive first pass metabolism. The combination with a gut-specific P-gp-inhibitor is a rational approach to improve bioavailability. Previous work has shown saturation at doses of paclitaxel above $300 \mathrm{mg} / \mathrm{m}^{2}$ with once daily dosing. Other P-gp inhibitors have been trialled, but have not progressed due to either toxicity of the inhibitor, or failure to achieve comparable exposure.

The classical MTD approach to drug development may not be ideal when developing oral versions of agents with well-established dosing schedules and known therapeutic effects. Several other studies have taken an MTD approach but have achieved saturation which may compromise therapeutic drug exposure.

We took a PK-directed approach to develop a schedule that could potentially achieve similar exposure, measured by AUC, as IV paclitaxel $80 \mathrm{mg} / \mathrm{m}^{2}$.

Unsurprisingly, the $\mathrm{C}_{\max }$ of paclitaxel with oPac+E is lower than with IV. However, with multi-day dosing we were able to demonstrate AUC of paclitaxel with oPac+E was comparable within the prespecified bounds compared to IV administration, meeting the study primary endpoint.

We found no difference in oral bioavailability between Asian and European patients, and PK profile was consistent after 4 weeks exposure to oPac $+\mathrm{E}$ suggesting that there is no clinically relevant induction of $\mathrm{P}$-gp or CYP enzymes. This implies that prolonged treatment will not result in meaningful reductions in drug exposure. 
The mean terminal half-life was longer with oral exposure than IV, suggesting either ongoing oral absorption, or that IV clearance differs, possibly mediated by cremophor.

Several challenges remain. This study was designed to measure PK profile and it is not possible to determine, from this study, the safety profile of prolonged administration. This is important as lower Cmax could impact on toxicity profile such as the incidence of alopecia, or the development of neuropathy. Similarly, time above a minimum inhibitory concentration may be relevant for both efficacy and toxicity. The time above a minimum inhibitory concentration may be different between IV and oral. The impact of this hypothesis on efficacy and toxicity cannot be established by the present study design and would require a randomised study comparing IV and oral administration, powered for efficacy and toxicity rather than PK exposure. Such studies are underway, and can be undertaken with confidence based on the present study results.

We found that $\mathrm{oPac}+\mathrm{E}$ was adequately tolerated in the three-day schedule but further study will be required to establish the full safety profile with repeat dosing.

Our study used strict fasting requirements with patients fasting 8 hours prior and 4 hours after administration. This provided ideal conditions for studying PK parameters however is unlikely to be feasible in routine clinical practice. Food studies are required.

The oral formulation also uses slightly less than 8 times the raw amount of paclitaxel for oral dosing compared to IV administration. This does have potential consequences for manufacture and supply for such a widely used anti-neoplastic.

The schedule we devised in this study has been taken forward for further study, which has recently completed. That study enrolled 402 patients with metastatic breast cancer and randomised patients in a 2:1 ratio to receive our schedule of $\mathrm{oPac}+\mathrm{E}$ or IV Paclitaxel at the registration dose of $175 \mathrm{mg} / \mathrm{m}^{2} \mathrm{Q} 3 \mathrm{~W}$. The Oral schedule achieved higher response rate (primary end-point) and numerically longer median OS (27.9 months v 16.9 months, study not powered for OS). Toxicities were much less in oral group, with only $1 \%$ of patients experiencing grade 3 polyneuropathy (PNP), versus $8 \%$ in the IV group (all grades PNP $17 \%$ versus 57\%). The much lower incidence and severity of neuropathy could be a major advantage in the treatment of cancer patients. Additionally, fewer patients experienced alopecia, but slightly more patients had GI disturbance or neutropenia. [25]

In conclusion, we found that a combination of oral paclitaxel $205 \mathrm{mg} / \mathrm{m} 2$ and encequidar $15 \mathrm{mg}$ in a threeday schedule is equivalent on AUC to paclitaxel $80 \mathrm{mg} / \mathrm{m}^{2}$ and provides advantages to cancer patients over traditional IV weekly paclitaxel. PK parameters after 4 weeks exposure were unchanged, indicating no evidence of induction of P-gp or CYP enzymes. There was no evidence of variation between Asian and European ethnicity. There were no concerning safety signals. Participants preferred oPac+E to IV paclitaxel. oPac+E may be a candidate to replace weekly IV paclitaxel subject to confirmatory safety and efficacy data.

\section{Acknowledgments}

We are grateful to the patients and their families who gave up significant time to advance scientific discovery and aid future patients.

\section{Author contributions}

Study design: CJ TH NH DC DK WC RK SD

Recruited and managed patients on study treatment: CJ ES PB BM NH KC TC MD HY SD

PK analysis: TH NH DC JZ WC RK

Analyzed data: all authors

Wrote manuscript: CJ SD ES HP DC WC

Approved manuscript: all authors 
All authors have had full access to PK and clinical trial data. All authors vouch for the accuracy of the data.

\section{Conflict of interest statement:}

DC, DK, JH, JZ, WC, RK are employees of Athenex INC, the sponsors of this study. CJ received travel costs to present an abstract of this paper at ASCO 2018. No other conflicts of interest to declare

Funding information: this study was funded by Athenex IN, Buffalo, NY.

\section{Data availability statement}

The data that support the findings of this study are available from the corresponding author upon reasonable request. Some data may not be made available because of privacy or ethical restrictions.

\section{References}

1. Ghersi D, Willson ML, Chan MMK, Simes J, Donoghue E, Wilcken N. Taxane-containing regimens for metastatic breast cancer. The Cochrane database of systematic reviews . 2015;2015(6):CD003366-CD003366. doi:10.1002/14651858.CD003366.pub3

2. Clamp AR, James EC, McNeish IA, et al. Weekly dose-dense chemotherapy in first-line epithelial ovarian, fallopian tube, or primary peritoneal carcinoma treatment (ICON8): primary progression free survival analysis results from a GCIG phase 3 randomised controlled trial. The Lancet . 2019;394(10214):2084-2095. doi:10.1016/s0140-6736(19)32259-7

3. Masters GA, Temin S, Azzoli CG, et al. Systemic Therapy for Stage IV Non-Small-Cell Lung Cancer: American Society of Clinical Oncology Clinical Practice Guideline Update.Journal of Clinical Oncology . 2015;33(30):3488-3515. doi:10.1200/jco.2015.62.1342

4. Lu Z, Zhang X, Liu W, et al. A multicenter, randomized trial comparing efficacy and safety of paclitaxel/capecitabine and cisplatin/capecitabine in advanced gastric cancer. Gastric Cancer . 2018;21(5):782791. doi:10.1007/s10120-018-0809-y

5. Rowinsky EK. Paclitaxel pharmacology and other tumor types. Semin Oncol. Dec 1997;24(6 Suppl 19):S19-1-s19-12.

6. De Bono JS, Oudard S, Ozguroglu M, et al. Prednisone plus cabazitaxel or mitoxantrone for metastatic castration-resistant prostate cancer progressing after docetaxel treatment: a randomised open-label trial. The Lancet . 2010;376(9747):1147-1154. doi:10.1016/s0140-6736(10)61389-x

7. Lee H, Park S, Kang JE, Lee HM, Kim SA, Rhie SJ. Efficacy and safety of nanoparticle-albumin-bound paclitaxel compared with solvent-based taxanes for metastatic breast cancer: A meta-analysis. Scientific Reports . 2020;10(1)doi:10.1038/s41598-019-57380-0

8. Wani MC, Taylor HL, Wall ME, Coggon P, McPhail AT. Plant antitumor agents. VI. Isolation and structure of taxol, a novel antileukemic and antitumor agent from Taxus brevifolia.Journal of the American Chemical Society . 1971;93(9):2325-2327. doi:10.1021/ja00738a045

9. Walsh V, Goodman J. The billion dollar molecule: Taxol in historical and theoretical perspective.Clio Med . 2002;66:245-67. doi:10.1163/9789004333499_013

10. Joerger M. Treatment regimens of classical and newer taxanes. Cancer Chemother Pharmacol . Feb 2016;77(2):221-33. doi:10.1007/s00280-015-2893-6

11. Schrag D, Hershman DL, Basch E. Oncology Practice During the COVID-19 Pandemic. JAMA . 2020;323(20):2005-2006. doi:10.1001/jama.2020.6236

12. Gosain R, Abdou Y, Singh A, Rana N, Puzanov I, Ernstoff MS. COVID-19 and Cancer: a Comprehensive Review.Curr Oncol Rep . May 8 2020;22(5):53. doi:10.1007/s11912-020-00934-7 
13. Jibodh RA, Lagas JS, Nuijen B, Beijnen JH, Schellens JH. Taxanes: old drugs, new oral formulations.Eur $J$ Pharmacol . Oct 5 2013;717(1-3):40-6. doi:10.1016/j.ejphar.2013.02.058

14. Schellens JHM, Malingre MM, Kruijtzer CMF, et al. Modulation of oral bioavailability of anticancer drugs: from mouse to man. 2000;12(2):103-110. doi:10.1016/s0928-0987(00)00153-6

15. Kartner N, Riordan JR, Ling V. Cell surface P-glycoprotein associated with multidrug resistance in mammalian cell lines. Science. Sep 23 1983;221(4617):1285-8. doi:10.1126/science.6137059

16. Helgason HH, Kruijtzer CMF, Huitema ADR, et al. Phase II and pharmacological study of oral paclitaxel (Paxoral) plus ciclosporin in anthracycline-pretreated metastatic breast cancer. 2006;95(7):794800. doi:10.1038/sj.bjc.6603332

17. van Asperen J, van Tellingen O, van der Valk MA, Rozenhart M, Beijnen JH. Enhanced oral absorption and decreased elimination of paclitaxel in mice cotreated with cyclosporin A. Clin Cancer Res . Oct 1998;4(10):2293-7.

18. Malingre MM, Terwogt JM, Beijnen JH, et al. Phase I and pharmacokinetic study of oral paclitaxel. $J$ Clin Oncol . Jun 2000;18(12):2468-75. doi:10.1200/jco.2000.18.12.2468

19. Paek IB, Ji HY, Kim MS, Lee G, Lee HS. Metabolism of a new P-glycoprotein inhibitor HM-30181 in rats using liquid chromatography/electrospray mass spectrometry. 2006;20(9):1457-1462. doi:10.1002/rcm.2468

20. Paek IB, Ji HY, Kim MS, Lee GS, Lee HS. Simultaneous determination of paclitaxel and a new Pglycoprotein inhibitor HM-30181 in rat plasma by liquid chromatography with tandem mass spectrometry. 2006;29(5):628-634. doi:10.1002/jssc.200500368

21. Kim T-E, Gu N, Yoon SH, et al. Tolerability and Pharmacokinetics of a New P-Glycoprotein Inhibitor, HM30181, in Healthy Korean Male Volunteers: Single- and Multiple-Dose Randomized, Placebo-Controlled Studies. 2012;34(2):482-494. doi:10.1016/j.clinthera.2012.01.003

22. Lee HJ, Heo D-S, Cho J-Y, et al. A Phase I Study of Oral Paclitaxel with a Novel PGlycoprotein Inhibitor, HM30181A, in Patients with Advanced Solid Cancer. 2014;46(3):234-242. doi:10.4143/crt.2014.46.3.234

23. Lee KW, Lee KH, Zang DY, et al. Phase I/II Study of Weekly Oraxol for the Second-Line Treatment of Patients With Metastatic or Recurrent Gastric Cancer. 2015;20(8):896-897. doi:10.1634/theoncologist.20150202

24. Jackson CGCA, Bayston KF, McLaren BR, et al. An open label, randomised cross-over bioavailability study of oral paclitaxel (oraxol) compared to intravenous paclitaxel $80 \mathrm{mg} / \mathrm{m} 2$. Journal of Clinical Oncology . 2016;34(15_suppl):2569-2569. doi:10.1200/JCO.2016.34.15_suppl.2569

25. Umanzor G, Cutler DL, Barrios FJ, et al. Abstract GS6-01: Oral paclitaxel with encequidar: The first orally administered paclitaxel shown to be superior to IV paclitaxel on confirmed response and survival with less neuropathy: A phase III clinical study in metastatic breast cancer. Cancer Research . 2020;80(4 Supplement):GS6-01. doi:10.1158/1538-7445.SABCS19-GS6-01 
Figure 1: Study consort diagram

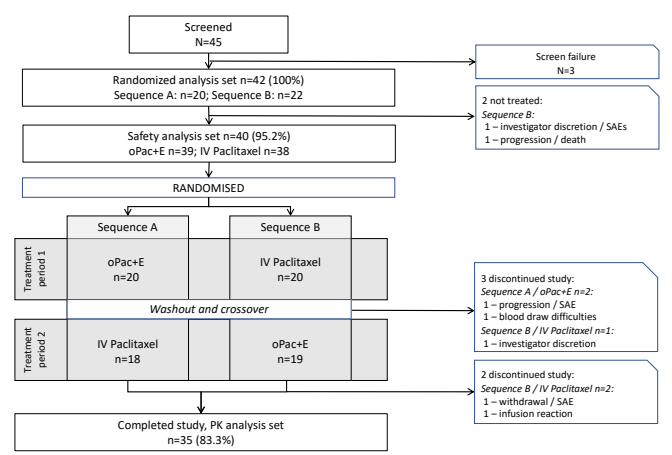



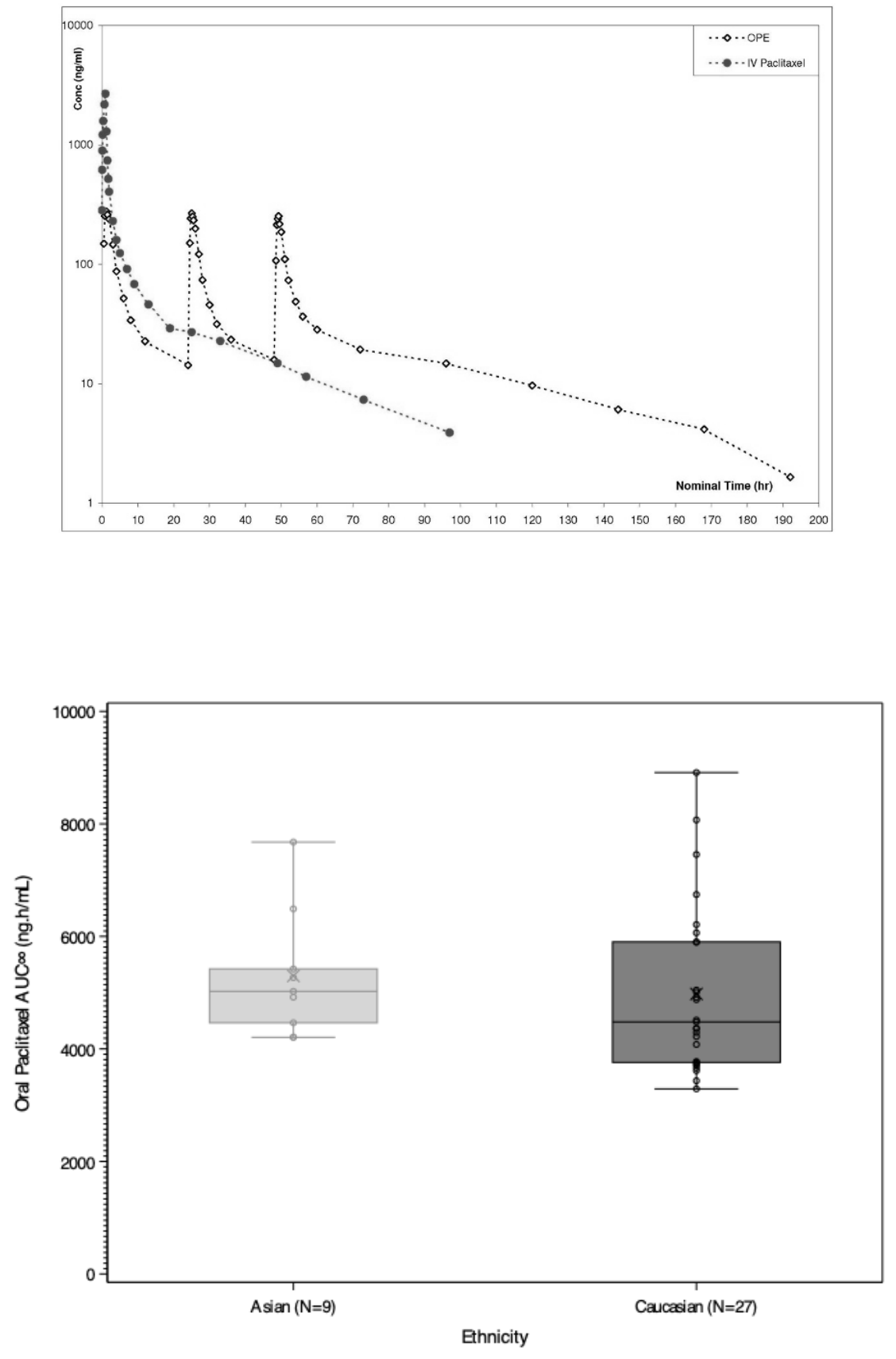

\section{Hosted file}

ORAX002 BJCP Manuscript Table_1.pdf available at https://authorea.com/users/366340/articles/ 500869-oral-paclitaxel-with-encequidar-compared-to-intravenous-paclitaxel-in-patientswith-advanced-cancer-a-randomised-crossover-pharmacokinetic-study

Hosted file 
ORAX002 BJCP Manuscript Table_2.pdf available at https://authorea.com/users/366340/articles/ 500869-oral-paclitaxel-with-encequidar-compared-to-intravenous-paclitaxel-in-patientswith-advanced-cancer-a-randomised-crossover-pharmacokinetic-study

\section{Hosted file}

ORAX002 BJCP Manuscript Table_3.pdf available at https://authorea.com/users/366340/articles/ 500869-oral-paclitaxel-with-encequidar-compared-to-intravenous-paclitaxel-in-patientswith-advanced-cancer-a-randomised-crossover-pharmacokinetic-study

\section{Hosted file}

ORAX002 BJCP Manuscript Table_4.pdf available at https://authorea.com/users/366340/articles/ 500869-oral-paclitaxel-with-encequidar-compared-to-intravenous-paclitaxel-in-patientswith-advanced-cancer-a-randomised-crossover-pharmacokinetic-study 